\title{
Comparison of the effects of exercise and anti-TNF treatment on cardiovascular health in rheumatoid arthritis: results from two controlled trials
}

\author{
Jet J. C. S. Veldhuijzen van Zanten ${ }^{1,2}\left(\mathbb{D}^{-} \cdot\right.$ Aamer Sandoo $^{3}\left(\mathbb{0} \cdot\right.$ George S. Metsios $^{2,4}(\mathbb{D})$ \\ Antonios Stavropoulos-Kalinoglou ${ }^{5}$ (D) Nikos Ntoumanis ${ }^{6}$ (D) George D. Kitas ${ }^{1,2}{ }^{(1)}$
}

Received: 22 August 2018 / Accepted: 20 October 2018 / Published online: 12 November 2018

(c) The Author(s) 2018

\begin{abstract}
People with rheumatoid arthritis (RA) are at increased risk for cardiovascular disease (CVD). Both pharmacological treatment and exercise are suggested in the management of CVD risk in RA. This study explored the effects of exercise and anti-TNF treatment on CVD risk in RA. Twenty RA patients (70\% female, 50 (10) years) completed a 3-month exercise intervention and 23 RA patients (65\% female, 54 (15) years) started anti-TNF treatment. Markers of disease activity, CVD risk, and vascular function were assessed before and after 3-months of intervention/treatment. Both exercise and anti-TNF treatment improved functional ability and fatigue, anti-TNF treatment was more successful in improving inflammation, disease activity, functional ability and pain. Exercise induced a reduction in overall CVD risk and improvement in vascular function, which was significantly different from anti-TNF treatment where no such changes were found. These findings showed that exercise and anti-TNF had differential effects on CVD risk in RA, and should be combined for optimal CVD risk reduction. Whereas anti-TNF treatment is likely to impact on CVD risk through reducing the systemic inflammatory load, exercise should be recommended to people with RA as an effective self-management strategy to reduce CVD risk further. Once RA patients have responded successfully to anti-TNF treatment, increasing exercise should be encouraged to reduce the risk for CVD. Thus, supporting exercise programmes when the disease is controlled, is likely to enhance the uptake and the maintenance of exercise, which will result in additional benefits to cardiovascular health and wellbeing in people with RA.
\end{abstract}

Keywords Rheumatoid arthritis $\cdot$ Exercise $\cdot$ Anti-TNF treatment $\cdot$ Cardiovascular disease risk

Preliminary analyses of the data presented in this manuscript have been presented at the Annual Conference of the British Society for Rheumatology: Veldhuijzen van Zanten JJCS, Metsios GS, Stavropoulos-Kalinoglou A, Sandoo A, Kitas GD (2013). Endothelial function in patients with rheumatoid arthritis: the effects of exercise and anti-TNF treatment. Rheumatology, 52:77.

Jet J. C. S. Veldhuijzen van Zanten

veldhujj@bham.ac.uk

1 School of Sport, Exercise and Rehabilitation Sciences, University of Birmingham, Birmingham B15 2TT, UK

2 Rheumatology Department, Dudley Group NHS Foundation Trust, Pensnett Road, Dudley DY1 2HQ, UK

3 School of Sport, Health and Exercise Sciences, Bangor University, George Building, Bangor LL57 2PZ, UK

\section{Background}

Rheumatoid arthritis (RA) is a chronic autoimmune disease which is characterised by high-grade systemic inflammation, swollen and painful joints [1]. The impact of RA is not restricted to the joints, patients often suffer from increased fatigue and depression [2], which influence psychological wellbeing. Patients with RA are also at increased risk

4 Faculty of Education Health and Wellbeing, University of Wolverhampton, Gorway Road, Walsall WS1 3BD, UK

5 Carnegie School of Sport, Leeds Beckett University, Cavendish 101, Headingley Campus, Leeds LS6 3QU, UK

6 School of Psychology, Curtin University, GPO Box U1987, Perth, WA 6845, Australia 
for cardiovascular disease (CVD) compared to the general population, with up to $50 \%$ of patients with RA dying as a result of cardiovascular complications [3]. The reasons for this increased risk are not fully understood, but both highgrade systemic inflammation and traditional CVD risk factors, which are prevalent in RA (e.g. hypertension [4] and obesity [4]), are thought to contribute to this [3, 6]. Indeed, a recent large international cohort study indicated that $70 \%$ of the CVD events could be attributed to CVD risk factors and RA-disease-related factors [7]. It is therefore not surprising that the European League against Rheumatism (EULAR) recommendations for CVD risk management in RA now include both control of disease activity through medication and CVD-risk reducing lifestyle interventions such as exercise [8].

Exercise is an effective lifestyle intervention to reduce CVD risk in the general population as well as in people with an already increased risk. Exercise can also reduce the risk for recurrence of cardiac events in those with established CVD $[9,10]$. Exercise can achieve these benefits through improvements in individual CVD risk factors, such as lowering blood pressure and improving lipid profile. However, the reduction in CVD risk cannot be explained solely by a reduction in these individual risk factors, the effects of exercise on the vasculature have been suggested to also contribute to the reduction in CVD risk [11]. Despite the abundance of information regarding the benefits of exercise for CVD risk in the general population, exercise has only recently been shown to improve overall CVD risk and endothelial function in RA [12, 13]. The potential of exercise to reduce CVD risk in RA is underscored by including exercise as a management strategy of CVD risk in RA by EULAR [8].

Anti-tumor necrosis factor- $\alpha$ (anti-TNF) is an effective treatment to reduce inflammation and overall disease activity in RA. There is strong evidence from meta-analyses that anti-TNF treatment is associated with a reduction in risk for acute cardiovascular events in RA [14, 15]. The exact mechanisms through which anti-TNF can improve CVD risk are not known. Even though the treatment specifically targets the cytokine TNF, a reduction in TNF reduces other inflammatory markers through various signalling pathways [16], which has been suggested to reduce the CVD risk $[1,14]$. Other factors which have been proposed to contribute to the reduced CVD risk include improvements in functional ability, less need for medication with an increased risk for CVD (e.g. corticosteroids [14]), and improvement in endothelial function [6].

In sum, exercise and pharmacological treatments effective in reducing inflammation have both been shown to reduce CVD risk in RA. However, it is not known how these different treatments compare to each other. Given the potential differences in mechanisms underlying changes following exercise and pharmacological treatment, it is likely that these interventions have differential effects. To our knowledge, the secondary analyses presented here are the first report comparing the effects of exercise with the effects of anti-TNF treatment on markers of disease activity, CVD risk factors, and vascular function in RA. Getting a better understanding of the effects of these different treatments can help inform clinical practice regarding effective strategies to reduce CVD risk in this population.

\section{Methods}

\section{Participants}

All participants were recruited from the Dudley Group NHS Foundation Trust Rheumatology outpatient clinics for two separate studies. Twenty participants were recruited to participate in an exercise intervention (Registered trial: ISRCTN50861407). Twenty-three participants who were about to start anti-TNF treatment on clinical indication were recruited into a 3-month prospective study. Both studies complied with the Declaration of Helsinki, were approved by local research ethics committees (exercise: Black Country Research Ethics Committee, anti-TNF: Birmingham East North and Solihull Research Ethics Committee), and all participants provided written informed consent. All participants met the RA diagnosis according to revised ACR criteria [17] and none of the participants had established CVD (e.g. had experienced cardiac event). Additional exclusion criteria for those in the exercise intervention were participating in structured exercise in the previous 6 months, comorbidities incompatible with exercise according to the American College of Sports Medicine [18]. All participants completed assessments at pre-intervention baseline and at three months. Detailed information regarding participants, interventions and assessments is provided elsewhere [19, 20].

\section{Exercise programme}

Participants in the exercise intervention completed an exercise tolerance test to determine their maximal aerobic capacity $\left(\mathrm{VO}_{2 \max }\right)$. The exercise programme was developed based on the participant's ability and preferences using apparatus such as treadmills, cycle, hand and rowing ergometers. Participants were asked to exercise three times per week for $1 \mathrm{~h}$. Two sessions of this individualised programme were conducted in a supervised setting and one unsupervised session at home. Each sessions started with 10 min warm up, followed 30-40 min where they completed three circuits at a heart rate corresponding to $70 \%$ of their $\mathrm{VO}_{2 \max }$, and ended with a 5-10 min cool down [19]. 


\section{Anti-TNF treatment}

Fifteen participants started on adalimumab, six participants on etanercept, and two participants on infliximab. None of the participants had previously received any other biologic drugs, including anti-TNF treatment. Participants did not change other medication during the course of the study [20]. Patients continued treatment with other drugs during the study period, including methotrexate (70\%), cyclooxygenase II inhibitors (4\%), nonsteroidal anti-inflammatory drugs (26\%), prednisolone (26\%), anti-hypercholesterolemic drugs $(18 \%)$, and antihypertensive drugs (22\%).

\section{Assessments}

\section{Disease activity}

A range of clinical and self-reported measures of disease activity was used. Disease activity was assessed with Disease Activity Score (DAS28 [21]), taking into account number of painful and swollen joints as well as overall health in the previous week. Functional ability was assessed with the Stanford Health Assessment Questionnaire (HAQ [22]). Systemic inflammation was assessed with high-sensitivity C-reactive protein (CRP), erythrocyte sedimentation rate (ESR) and fibrinogen using the standardised laboratory protocols. Fatigue was assessed with the multidimensional assessment of fatigue [23]. Participants were asked to rate their overall pain in the previous week using a visual analogue scale ranging from 0 'no pain at all' to 100 'worst pain ever'.

\section{Cardiovascular risk measures}

Blood pressure and serological markers of CVD risk including cholesterol, triglycerides, high-density lipoproteins (HDL) and insulin sensitivity (homeostasis model assessment-HOMA and Quantitative insulin-sensitivity check index-QUICKI), were assessed. Global CVD risk was estimated using the Framingham risk score [24].

\section{Vascular function}

Large vessel endothelium-dependent [flow-mediated dilatation (FMD)] and endothelium-independent [sublingual glyceryl-trinitrate (GTN)] function was assessed using high-resolution ultrasonography. Laser Doppler imaging (Moor LDI 2 SIM: Moor Instruments, Ltd Devon, UK) with iontophoresis of acetylcholine (ACh) and sodium-nitroprusside (SNP) was used to measure endothelium-dependent and endothelium-independent microvascular function, respectively [25].

\section{Statistical analyses}

Mixed linear modeling was used to explore within- and between participant changes in markers of disease activity, CVD risk and vascular function separately. This approach was chosen over repeated measures ANOVA as it does not assume sphericity of the data, can process non-normally distributed data and deal with occasional missing data [26]. The within-person level was Time (level 1) and between-person level was participant (level 2). Group (anti-TNF and exercise) was a predictor variable at level 2 , with anti-TNF as the reference group. Time by Group interaction effects were calculated to explore differences in changes over time between groups. Sex and mean centered age were entered at level 2 as covariates. In interpreting the findings, it is worth noting that the Time $\times$ Group interactions test whether the rate of change from baseline to 3 months was equivalent across the two groups for a female of average age. All analyses were conducted using IBM SPSS, version 22.

\section{Results}

\section{Participant characteristics at baseline}

There were no significant differences between the groups with regards to age (exercise: 50 [10], anti-TNF: 54 [15] years), disease duration (exercise: 6 [1-25], anti-TNF: 7 [2-43] years) or sex (exercise: 70\% women, anti-TNF: 65\% women). Baseline levels of markers of disease activity, CVD risk factors, and vascular function are reported in Table 1. As expected, participants starting anti-TNF had higher baseline levels of disease activity which reached significance for DAS28 and fibrinogen. In addition, baseline levels of functional ability, fatigue and pain were also poorer in participants in the anti-TNF group. CVD risk indicators were not significantly different, but large vessel endotheliumindependent function was poorer in the exercise group at baseline.

\section{Effects of exercise and anti-TNF treatment}

The effects of exercise and anti-TNF treatment are presented in Table 1. Anti-TNF treatment was successful at improving disease-related markers, whereas after 3 months of exercise only functional ability and fatigue were improved. Significant Time by Group interaction effects were found for Fibrinogen, DAS28, HAQ, and pain, indicating that antiTNF treatment induced greater improvements in these measures than exercise. Given the baseline differences in most of the disease-related measures, it is important to highlight that 
Table 1 Estimated means (SE) for baseline and 3 months for the exercise group and the anti-TNF group

\begin{tabular}{|c|c|c|c|c|c|}
\hline & \multicolumn{2}{|l|}{ Exercise } & \multicolumn{2}{|l|}{ Anti-TNF } & \multirow[t]{2}{*}{ Time $\times$ group interaction } \\
\hline & Baseline & 3 months & Baseline & 3 months & \\
\hline \multicolumn{6}{|l|}{ Disease related measures } \\
\hline C-reactive protein (mg/l) & $6.96(3.22)$ & $3.30(4.12)$ & $13.05(2.39)$ & $7.15(2.70)^{*}$ & $t(41.5)=0.54, p=.590$ \\
\hline Erythrocyte Sedimentation Rate (mmhr) & $19.68(6.38)$ & $18.86(7.37)$ & $27.21(4.65)$ & $27.88(4.81)$ & $t(38.7)=-0.20, p=.841$ \\
\hline Fibrinogen $(\mathrm{g} / \mathrm{L})$ & $4.11(0.29)$ & $4.02(0.27)$ & $5.05(0.21)^{\#}$ & $4.33(0.17)^{*}$ & $t(36.1)=2.36, p=.024$ \\
\hline DAS28 & $3.33(0.34)$ & $2.93(0.33)$ & $4.31(0.26)^{\#}$ & $2.92(0.26)^{*}$ & $t(39.6)=3.02, p=.004$ \\
\hline HAQ & $1.41(0.23)$ & $1.27(0.19)^{*}$ & $2.12(0.17)^{\#}$ & $1.30(0.13)^{*}$ & $t(37.9)=2.41, p=.021$ \\
\hline Fatigue & $22.31(3.86)$ & $15.54(3.92)^{*}$ & $35.78(2.85)^{\#}$ & $21.72(2.60)^{*}$ & $t(38.6)=1.86, p=.071$ \\
\hline Pain (out of 100) & $36.59(8.85)$ & $37.82(9.78)$ & $64.00(6.49)^{\#}$ & $37.72(6.50)^{*}$ & $t(38.5)=2.81, p=.008$ \\
\hline \multicolumn{6}{|l|}{ CVD risk factors } \\
\hline Body mass index $\left(\mathrm{kg} / \mathrm{m}^{2}\right)$ & $28.81(1.77)$ & $28.64(0.29)$ & $30.22(1.41)$ & $30.34(0.20)$ & $t(38.0)=21.39, p=.324$ \\
\hline Framingham risk score ${ }^{\mathrm{a}}$ & $5.50(1.87)$ & $4.05(0.69)^{*}$ & $5.79(1.34)$ & $5.74(0.50)$ & $t(36.1)=-2.04, p=.049$ \\
\hline Systolic blood pressure (mm Hg) & $130.61(3.90)$ & $125.81(3.40)$ & $126.88(2.98)$ & $119.19(2.32)$ & $t(41.0)=0.85, p=.401$ \\
\hline Diastolic blood pressure $(\mathrm{mm} \mathrm{Hg})$ & $77.96(2.24)$ & $75.06(2.06)$ & $79.15(1.71)$ & $74.08(1.40)^{*}$ & $t(41.0)=1.05, p=.298$ \\
\hline Cholesterol (mmol/L) & $5.17(0.32)$ & $5.12(0.18)$ & $4.88(0.24)$ & $4.97(0.11)^{*}$ & $t(36.0)=-0.82, p=.417$ \\
\hline Triglycerides (mmol/L) & $1.31(0.22)$ & $1.26(0.22)$ & $1.27(0.17)$ & $1.48(0.14)$ & $t(38.0)=-1.18, p=.245$ \\
\hline $\mathrm{HDL}(\mathrm{mmol} / \mathrm{L})$ & $1.50(0.09)$ & $1.53(0.05)$ & $1.56(0.07)$ & $1.55(0.03)$ & $t(36.7)=0.81, p=.424$ \\
\hline HOMA & $1.79(1.22)$ & $2.76(1.50)$ & $2.59(0.79)$ & $1.97(0.92)$ & $t(34.8)=1.06, p=.295$ \\
\hline QUICKI & $0.34(0.01)$ & $0.34(0.01)$ & $0.35(0.01)$ & $0.34(0.01)$ & $t(26.9)=0.87, p=.867$ \\
\hline \multicolumn{6}{|l|}{ Vascular function } \\
\hline Large vessel endothelium-dependent (FMD\%) & $7.55(2.24)$ & $18.34(2.59)^{*}$ & $8.93(1.62)$ & $11.50(1.67)$ & $t(37.7)=3.17, p=.003$ \\
\hline Large vessel endothelium-independent (GTN\%) & $18.81(2.18)$ & $27.11(2.01)^{*}$ & $23.43(1.60)^{\#}$ & $24.67(1.34)$ & $t(35.9)=3.51, p=.001$ \\
\hline Microvascular endothelium-dependent (ACh\%) & $331(72)$ & $426(83)$ & $301(50)$ & $331(49)$ & $t(34.1)=0.79, p=.437$ \\
\hline Microvascular endothelium-independent (SNP\%) & $239(57)$ & $434(52)^{*}$ & $230(41)$ & $239(31)$ & $t(34.4)=3.57, p=.001$ \\
\hline
\end{tabular}

All values are estimated means for a female with average age. All analyses were conducted with age and sex as covariates

$D A S 28$ disease activity score in 28 joints, $H A Q$ health assessment questionnaire, $H D L$ high density lipids, $H O M A$ homeostasis model assessment, QUICKI quantitative insulin-sensitivity check index, FMD flow-mediated dilatation, GTN glyceryl trinitrate, ACh acetyl choline, SNP sodium nitroprusside

*: significantly different from baseline

\#: significantly different from baseline exercise

${ }^{a}$ no covariates used for the analyses

after 3 months of anti-TNF treatment disease-related markers were similar to the baseline values of the exercise group.

Anti-TNF treatment did not induce many changes in CVD risk factors, with the exception of an increase in cholesterol and a reduction in diastolic blood pressure. In contrast, exercise induced a reduction in Framingham risk score and improvement in vascular function, which were not changed in response to anti-TNF treatment (significant Time by Group interaction effects, $p$ 's $<0.05$, see Table 1 ).

\section{Discussion}

The current study showed that exercise and anti-TNF treatment had differential effects on CVD risk in people with RA. Exercise was more successful at improving overall CVD risk and vascular function compared to anti-TNF treatment in RA. Whereas both exercise and anti-TNF treatment improved functional ability and fatigue, anti-TNF treatment was more successful in improving inflammation, DAS28, functional ability and pain.

At baseline, people starting anti-TNF treatment had higher disease activity, fatigue and pain as well as lower functional ability compared to the participants starting the exercise programme. This finding is not surprising, given that anti-TNF treatment is recommended by NICE only if patients experience high disease activity and have not responded to a combination of conventional disease-modifying antirheumatic drugs. We also know from our own experience that RA patients who are volunteering to participate in an exercise study tend to have relatively well controlled disease activity. The improvements in disease-related measures following anti-TNF provide evidence for the effectiveness of this treatment in controlling disease activity. As 
participants starting anti-TNF treatment had higher levels of disease activity, there was also more opportunity for improvement in this group, which could contribute to the significant Time by Group interaction effects. Indeed, following anti-TNF treatment, disease-related measures were similar compared to the baseline levels of the patients in the exercise intervention. It is important to note that most disease-related measures also improved with exercise, however, these improvements were only statistically significant for functional ability and fatigue. Both fatigue and functional ability are commonly mentioned barriers to exercise in people with RA [27], however, in line with previous research $[28,29]$, these findings show that exercise can improve these important patient-focussed measures. Thus, it is important for exercise programmes for people with RA to include education about the health benefits of exercise as well as support to overcome barriers to exercise.

Despite no significant changes in individual risk factors, global CVD risk was significantly reduced following exercise compared to anti-TNF treatment. Most individual risk factors showed an improvement following exercise, albeit not statistically significant. Together, these changes resulted in an improvement of global CVD risk, and provide evidence that only 3 months of exercise already results in a significant reduction in the risk for cardiovascular disease in RA. Given that anti-TNF treatment has been shown to reduce the risk for acute cardiovascular events $[14,15]$, the lack of changes in global CVD risk following anti-TNF was not expected. Greater CVD risk reduction has been associated with longer duration of anti-TNF treatment [30]. Thus, it is possible that 3 months of treatment is not sufficient to reduce global CVD risk, and longer term treatment is needed. Another possibility is that the CVD risk reduction following anti-TNF treatment is due to a reduction in disease-related inflammation, which is not incorporated in the global CVD risk calculator used in the current study.

The benefits of exercise on the vasculature are wellknown, and it has been suggested that the increases in shear stress during regular exercise enhance structural changes as well as signalling pathways and inflammatory processes within the vascular wall associated with vascular function $[11,31,32]$. Less is known about the effects of anti-TNF treatment on the vasculature. In vivo assessments of vascular function following successful treatment provide no strong evidence for direct associations between systemic inflammation and vascular function [33]. Vascular wall inflammation was significantly higher in those treated with anti-TNF compared to DMARDs, despite both groups achieving lowgrade systemic inflammation indicative of remission [34]. This discrepancy between local and systemic effects of TNF on vascular function has also been reported in experimental studies [35]. Thus, it is likely that the improvements in in vivo vascular function following exercise are due to lower levels of vascular wall inflammation, which is not achieved following anti-TNF treatment, despite an anti-TNF-induced reduction in systemic inflammation.

The differential benefits of exercise and anti-TNF of this study have important implications for the timing of encouragement for people with RA to become more physically active to reduce their CVD risk. Pain, fatigue and functional ability are commonly mentioned barriers to exercise and physical activity [27]. It is therefore not surprising that people with RA have indicated that once their disease activity is controlled, they are more likely to engage with behavioural interventions to reduce CVD risk, such as exercise [36-38]. Observational studies show that following successful treatment of disease activity in RA self-reported physical activity was increased [39] and medication-induced improvements in disease activity were related to physical activity increases [40]. Thus, providing support by offering exercise programmes when the disease is controlled, is likely to enhance the uptake and the maintenance of exercise, which as the current study reveals will result in additional benefits to their CVD risk and vascular function, as well as functional ability and fatigue.

The baseline differences between the two groups are a limitation of the current study, a randomised controlled trial would allow for a more stringent comparison between exercise and anti-TNF treatment. However, for ethical reasons, it is not possible to withhold pharmacological treatment from those who need it based on clinical assessment. It should also be acknowledged that the interventions were exclusive; participants either started exercise intervention or anti-TNF treatment and it is therefore not known what the effect would be of starting exercise and anti-TNF treatment simultaneously. The relatively brief follow-up period could be an explanation for the lack of effects of anti-TNF on CVD risk, particularly given that CVD risk reduction is related to the duration of the exposure to anti-TNF [30]. Nevertheless, other studies have reported transient improvements in endothelial function and some CVD risk factors following anti-TNF [33]. Even though endothelium-dependent microvascular function improved following exercise, this did not reach statistical significance, which could be due to some missing data for this measurement. Therefore, this will need to be confirmed in a larger cohort.

In summary, exercise and anti-TNF treatment have differential effects on cardiovascular health in people with RA. Exercise induced a reduction in overall CVD risk and improvement in vascular function, while anti-TNF treatment was better at improving disease-related measures. Thus, whereas anti-TNF treatment is likely to impact on CVD risk through reducing the systemic inflammatory load, exercise should be recommended to people with RA as an effective self-management strategy to reduce CVD risk further. Given that both disease-related factors and CVD risk factors 
have been shown to contribute to CVD events in RA [7], the combination of exercise and anti-TNF treatment might provide the best protection for CVD in RA. Pain, fatigue and functional ability are commonly mentioned barriers to exercise and physical activity [27], and observational studies already showed increases in physical activity following successful treatment of disease activity [39, 40]. Thus, supporting exercise programmes for people with RA when the disease is controlled, is likely to enhance the uptake and the maintenance of exercise, which will result in additional benefits for cardiovascular health and wellbeing.

Acknowledgements This work was supported by the Dudley Group R\&D Directorate cardiovascular programme and a University of Birmingham PhD student grant. The authors would like to acknowledge all of the participants who gave their time to participate in the study. In addition, we would like to thank the staff at the Clinical Research Unit and Russells Hall Hospital who provided assistance with data collection.

Author contributions JJCSVvZ, AS, GDK: design, recruitment, assessments, and data management of anti-TNF study. JJCSVvZ, GSM, ASK, GDK: design, recruitment, assessments, and data management of exercise study. JJCSVvZ, NN: analyses. All authors contributed to manuscript preparation.

Funding This study was funded by the Dudley Group R\&D Directorate cardiovascular programme and a University of Birmingham $\mathrm{PhD}$ student grant.

\section{Compliance with ethical standards.}

Conflict of interest Dr Veldhuijzen van Zanten declares that she has no conflict of interest, Dr Sandoo declares that he has no conflict of interest, Prof Metsios declares that he has no conflict of interest, Dr Stavropoulos-Kalinoglou declares that he has no conflict of interest, Prof Ntoumanis declares that he has no conflict of interest, and Prof Kitas declares that he has no conflict of interest.

Ethical approval All procedures performed in studies involving human participants were in accordance with the ethical standards of the institutional and national research committee and with the 1964 Helsinki declaration and its later amendments or comparable ethical standards. Informed consent: Informed consent was obtained from all individual participants included in the study.

Open Access This article is distributed under the terms of the Creative Commons Attribution 4.0 International License (http://creativeco mmons.org/licenses/by/4.0/), which permits unrestricted use, distribution, and reproduction in any medium, provided you give appropriate credit to the original author(s) and the source, provide a link to the Creative Commons license, and indicate if changes were made.

\section{References}

1. McInnes IB, Schett G (2017) Pathogenetic insights from the treatment of rheumatoid arthritis. Lancet 389:2328-2337
2. Gettings L (2010) Psychological well-being in rheumatoid arthritis: a review of the literature. Musculoskelet Care 8:99-106

3. Kitas GD, Gabriel SE (2011) Cardiovascular disease in rheumatoid arthritis: state of the art and future perspectives. Ann Rheum Dis 70:8-14

4. Panoulas VF, Metsios GS, Pace AV, John H, Treharne GJ, Banks MJ et al (2008) Hypertension in rheumatoid arthritis. Rheumatology 47:1286-1298

5. Stavropoulos-Kalinoglou A, Metsios GS, Koutedakis Y, Kitas GD (2011) Obesity in rheumatoid arthritis. Rheumatology 50:450-462

6. Greenberg JD, Furer V, Farkouh ME (2012) Cardiovascular safety of biologic therapies for the treatment of RA. Nat Rev Rheumatol 8:13-21

7. Crowson CS, Rollefstad S, Ikdahl E, Kitas GD, Van Riel PLCM, Gabriel SE et al (2018) Impact of risk factors associated with cardiovascular outcomes in patients with rheumatoid arthritis. Ann Rheum Dis 77:48-54

8. Agca R, Heslinga SC, Rollefstad S, Heslinga M, McInnes IB, Peters MJ et al (2017) EULAR recommendations for cardiovascular disease risk management in patients with rheumatoid arthritis and other forms of inflammatory joint disorders: 2015/2016 update. Ann Rheum Dis 76:17-28

9. Varghese T, Schultz WM, McCue AA, Lambert CT, Sandesara PB, Eapen DJ et al (2016) Physical activity in the prevention of coronary heart disease: implications for the clinician. Heart 102:904-909

10. Eijsvogels TM, Molossi S, Lee DC, Emery MS, Thompson PD (2016) Exercise at the extremes: the amount of exercise to reduce cardiovascular events. J Am Coll Cardiol 67:316-329

11. Wilson MG, Ellison GM, Cable NT (2015) Basic science behind the cardiovascular benefits of exercise. Heart 101:758-765

12. Stavropoulos-Kalinoglou A, Metsios GS, Veldhuijzen van Zanten JJCS, Nightingale P, Kitas GD, Koutedakis Y. Individualised aerobic and resistance exercise training improves cardiorespiratory fitness and reduces cardiovascular risk in patients with rheumatoid arthritis. Ann Rheum Dis 2013;72:1819-1825

13. Metsios GS, Stavropoulos-Kalinoglou A, Veldhuijzen van Zanten JJCS, Nightingale P, Sandoo A, Dimitroulas T et al (2013) Individualised exercise improves endothelial function in patients with rheumatoid arthritis. Ann Rheum Dis 73:748-751

14. Barnabe C, Martin B-J, Ghali WA (2011) Systematic review and meta-analysis: anti-tumor necrosis factor alpha therapy and cardiovascular events in rheumatoid arthritis. Arthr Care Res (Hoboken) 63:522-529

15. Roubille C, Richer V, Starnino T, McCourt C, McFarlane A, Fleming $\mathrm{P}$ et al (2015) The effects of tumour necrosis factor inhibitors, methotrexate, non-steroidal anti-inflammatory drugs and corticosteroids on cardiovascular events in rheumatoid arthritis, psoriasis and psoriatic arthritis: a systematic review and meta-analysis. Ann Rheum Dis 74:480-489

16. Udalova I, Monaco C, Nanchahal J, Feldmann M (2016) AntiTNF therapy. Microbiol Spectr 4(4):MCHD-0022-2015

17. Arnett FC, Edworthy SM, Bloch DA, McShane DJ, Fries JF, Cooper NS et al (1988) The American Rheumatism Association 1987 revised criteria for the classification of rheumatoid arthritis. Arthr Rheum 31:315-324

18. (2005) ACSM guidelines for exercise testing and prescription, 7th edn. Lippincott Williams \& Wilkins, Philadelphia, PA

19. Stavropoulos-Kalinoglou A, Metsios GS, Veldhuijzen van Zanten JJCS, Nightingale P, Kitas GD, Koutedakis Y (2013) Individualised aerobic and resistance exercise training improves cardiorespiratory fitness and reduces cardiovascular risk in patients with rheumatoid arthritis. Ann Rheum Dis 72:1819-1825 
20. Sandoo A, Kitas GD, Carroll D, Veldhuijzen van Zanten JJCS (2012) The role of inflammation and cardiovascular disease risk on microvascular and macrovascular endothelial function in patients with rheumatoid arthritis: a cross-sectional and longitudinal study. Arthr Res Ther 14:R117

21. Prevoo ML, Van 't Hof MA, Kuper HH, Van Leeuwen MA, Van de Putte LB, Van Riel PL (1995) Modified disease activity scores that include twenty-eight-joint counts. Development and validation in a prospective longitudinal study of patients with rheumatoid arthritis. Arthr Rheum 38:44-48

22. Zigmond AS, Snaith RP (1983) The hospital anxiety and depression scale. Acta Psychiatr Scand 67:361-370

23. Belza BL, Henke CJ, Yelin EH, Epstein WV, Gilliss CL (1993) Correlates of fatigue in older adults with rheumatoid arthritis. Nurs Res 42:93-99

24. Wilson PWF, D'Agostino RB, Levy D, Belanger AM, Silbershatz H, Kannel WB (1998) Prediction of coronary heart disease using risk factor categories. Circulation 97:1837-1847

25. Sandoo A, Kitas GD (2015) Methodological approach to noninvasive assessments of vascular function and morphology. J Vis Exp 96:e52339

26. Singer JD, Willett JB (2003) Applied longitudinal data analysis: modeling change and event occurrence. Oxford University Press, New York

27. Veldhuijzen van Zanten JJCS, Rouse PC, Hale ED, Ntoumanis N, Metsios GS, Duda JL et al (2015) Perceived barriers, facilitators and benefits for regular physical activity and exercise in patients with rheumatoid arthritis: a review of the literature. Sports Med 45:1401-1412

28. Metsios GS, Stavropoulos-Kalinoglou A, Veldhuijzen van Zanten JJCS, Treharne GJ, Panoulas VF, Douglas KM et al (2008) Rheumatoid arthritis, cardiovascular disease and physical exercise: a systematic review. Rheumatology 47:239-248

29. Rongen-van Dartel SA, Repping-Wuts H, Flendrie M, Bleijenberg G, Metsios GS, van den Hout WB et al (2015) Effect of aerobic exercise training on fatigue in rheumatoid arthritis: a meta-analysis. Arthr Care Res (Hoboken) 67:1054-1062

30. Nurmohamed M, Bao Y, Signorovitch J, Trahey A, Mulani P, Furst DE (2015) Longer durations of antitumour necrosis factor treatment are associated with reduced risk of cardiovascular events in patients with rheumatoid arthritis. RMD Open 1:e000080
31. Newcomer SC, Thijssen DH, Green DJ (1985) Effects of exercise on endothelium and endothelium/smooth muscle cross talk: role of exercise-induced hemodynamics. J Appl Physiol 111:311-320 2011

32. Metsios GS, Stavropoulos-Kalinoglou A, Sandoo A, Veldhuijzen van Zanten JJCS, Toms TE, John H et al (2010) Vascular function and inflammation in rheumatoid arthritis: the role of physical activity. Open Cardiovasc Med J 4:89-96

33. Sandoo A, Veldhuijzen van Zanten JJCS, Metsios GS, Carroll D, Kitas GD (2011) Vascular function and morphology in rheumatoid arthritis: a systematic review. Rheumatology 50:2125-2139

34. Moens SJ, van der Valk FM, Strang AC, Kroon J, Smits LP, Kneepkens EL et al (2016) Unexpected arterial wall and cellular inflammation in patients with rheumatoid arthritis in remission using biological therapy: a cross-sectional study. Arthr Res Ther $18: 115$

35. Cardillo C, Schinzari F, Mores N, Mettimano M, Melina D, Zoli A et al (2006) Intravascular tumor necrosis factor alpha blockade reverses endothelial dysfunction in rheumatoid arthritis. Clin Pharmacol Ther 80:275-281

36. John H, Hale ED, Treharne GJ, Carroll D, Kitas GD (2009) 'All singing from the same hymn sheet': healthcare professionals' perceptions of developing patient education material about the cardiovascular aspects of rheumatoid arthritis. Musculoskelet Care 7:256-271

37. John H, Hale ED, Treharne GJ, Carroll D, Kitas GD (2009) 'Extra information a bit further down the line': rheumatoid arthritis patients' perceptions of developing educational material about the cardiovascular disease risk. Musculoskelet Care 7:272-287

38. Withall J, Haase AM, Walsh NE, Young A, Cramp F (2016) Physical activity engagement in early rheumatoid arthritis: a qualitative study to inform intervention development. Physiotherapy 102:264-271

39. Konijn NP, Van Tuyl LH, Boers M, Den Uyl D, Ter Wee MM, Kerstens P et al (2016) Effective treatment for rapid improvement of both disease activity and self-reported physical activity in early rheumatoid arthritis. Arthr Care Res (Hoboken) 68:280-284

40. Prioreschi A, Hodkinson B, Tikly M, McVeigh JA (2014) Changes in physical activity measured by accelerometry following initiation of DMARD therapy in rheumatoid arthritis. Rheumatology 53:923-926 